\title{
Olfactory and gustatory abnormalities in COVID-19 cases
}

\author{
Fazilet Altin ${ }^{1} \cdot$ Cemal Cingi ${ }^{2} \cdot$ - Tankut Uzun ${ }^{2} \cdot$ Cengiz Bal ${ }^{3}$
}

Received: 16 May 2020 / Accepted: 17 June 2020 / Published online: 23 June 2020

(c) Springer-Verlag GmbH Germany, part of Springer Nature 2020

\begin{abstract}
Purpose At the time of writing, there is a pandemic affecting virtually every country on Earth. There is considerable discussion amongst clinicians as well as lay people about anosmia and ageusia in COVID-19 sufferers. We aimed to report the results from comprehensive olfactory and gustatory testing in a series of hospital in-patients.

Methods The prospective study evaluated 81 individuals with a COVID-19 infection, as confirmed by 2019 n-cov Real-Time PCR laboratory testing. The control group consisted of forty individuals with COVID-19 negative. Olfactory and gustatory testings were carried out by an examiner utilizing stringent safety standards and wearing full personal protective equipment. The results obtained in the case group were then compared with those obtained for the controls.

Results In the case group, 41(50.6\%) of patients were male and 40 (49.4\%) were female, mean age of 54.16 \pm 16.98 years (1895). In the control group, 21 (52.5\%) of subjects were male and 19 (47.5\%) were female, and mean age was $55 \pm 15.39$ years (18-90). Fifty (61.7\%) COVID-19-positive patients had complaints related to olfaction. The distribution of olfactory symptoms in the case group differed at the level of statistical significance from the control group $(p<0.001)$. Turning to gustatory abnormalities, within the case group, 22 individuals (27.2\%) had taste malfunction. A statistically significant difference was found in the distribution of gustatory abnormalities between cases and controls $(p<0.001)$.

Conclusions Olfactory and gustatory dysfunctions are strongly associated with SARS-CoV-2 infection. Hyposmia with or without hypogeusia is potentially a reliable indicator of latent COVID-19.
\end{abstract}

Keywords COVID-19 $\cdot$ Smell $\cdot$ Smell test $\cdot$ Taste $\cdot$ Virus infection

Cemal Cingi

ccingi@gmail.com

Fazilet Altin

drfaziletaltin@gmail.com

Tankut Uzun

uzuntankut@gmail.com

Cengiz Bal

cengiz@ogu.edu.tr

1 Department of Otorhinolaryngology, University of Health Sciences, Haseki Training and Research Hospital, Istanbul, Turkey

2 Medical Faculty, ENT Department, Eskişehir Osmangazi University, Eskişehir, Turkey

3 Medical Faculty, Biostatistics Department, Eskişehir Osmangazi University, Eskişehir, Turkey

\section{Introduction}

Coronaviruses are capable of producing a wide range of systemic infective diseases, both in humans and animals. In humans, these pathogens can produce the full spectrum of respiratory infective disease, from mild coryza to severe or fatal pneumonia [1]. Two coronaviruses of high pathogenic potential in humans have been responsible for widespread epidemics or pandemics within the last 20 years: Severe Acute Respiratory Syndrome CoV (SARS-CoV) and Middle East Respiratory Syndrome CoV (MERS-CoV) [2, 3]. Severe acute respiratory syndrome coronavirus 2 (SARS$\mathrm{CoV}-2$ ) is a beta coronavirus within group $2 \mathrm{~b}$ of the coronaviruses. It possesses around $70-80 \%$ genetic similarity with SARS-CoV [4]. COVID-19 is a novel coronavirus infective syndrome due to SARS-CoV-2 which has caused widespread fatalities on a global scale [5, 6]. SARS-CoV-2 has been noted as the 7th pathogenic coronavirus variant in humans and the 3rd responsible for a severe clinical syndrome, following SARS-CoV and MERS-CoV [7, 8]. 
There is wide variety in the clinical presentation of COVID-19 infection, on a spectrum from asymptomatic cases through mild to severe disease, which may or may not be accompanied by pneumonia [9]. A number of studies reveal that characteristic signs of COVID-19 include pyrexia, coughing, diarrhoea and lethargy [10]. Lately, it has been noted by numerous researchers that hyposmia or anosmia plus hypogeusia or ageusia (reduced or absent taste sensation) may occur. Acute loss of smell and taste was observed in $15.3 \%$ in the early stage of COVID-19-positive patients by Lee at al [11]. They also found that a higher prevalence in younger and female patients. They also declared that anosmia and ageusia may occur as the first symptom of COVID-19.

At the time of writing, there is a pandemic affecting virtually every country on Earth. There is considerable discussion amongst clinicians as well as lay people about anosmia and ageusia in COVID-19 sufferers. However, to the best of the authors' knowledge, a systematic evaluation of olfactory and gustatory testing in such individuals has yet to be published. In this paper, we report the results from comprehensive olfactory and gustatory testing in a series of hospital in-patients with viral lower and upper respiratory infections in whom COVID-19 was confirmed by PCR (polymerase chain reaction).

\section{Materials and methods}

\section{Study design and study groups}

This prospective study was undertaken at two centres: Eskisehir Osmangazi University, Medical Faculty, ENT Department and HSU Haseki Trainining and Research Hospital, ENT Department. Both centres are hospitals designated by the Turkish state for the treatment of patients suffering from COVID-19. The study evaluated a consecutive series of patients between 25 March 2020 and 20 April 2020. All the patients enrolled in the study were diagnosed with COVID-19, following criteria used by the WHO interim guidance documentation [12].

Eighty-one hospitalized patients were included in the analysis. In all such cases, SARS-CoV-2 was confirmed by laboratory testing. The presence of COVID-19 was considered confirmed where high-throughput sequencing or realtime reverse-transcription polymerase chain reaction analysis of throat and nasopharyngeal swab specimens produced a positive result. After collection, swab samples were put into a collection tube with a viral preservative solution. Confirmation of SARS-CoV-2 infection depended on real-time reverse-transcription polymerase chain reaction assay utilizing a SARS-CoV-2 nucleic acid detection kit (2019 n-cov
Real-Time PCR) with adherence to the protocol supplied by the kit manufacturer.

Prior to enrolment, either the participants themselves or a suitable relative in incapacitated patients gave consent to enter the study. Informed consent was obtained in written form for all participants, both cases and controls. The principles contained in the Declaration of Helsinki were followed throughout. The Ethics Committee Approval was obtained from Turkish Ministry of Health with the file number 2020-05-01T15_12_20.

\section{Data collection}

The study involved both a case and control group. Eightyone individuals formed the case group. These individuals all had a COVID-19 infection, as confirmed by 2019 n-cov Real-Time PCR laboratory testing. They were all questioned for any previous smell and taste disorders. Patients with previous history of smell and taste disorder were excluded from the study. All COVID-19 positive patients without any previous smell and/or taste disorders were included in this study group. The control group consisted of forty healthy individuals having the same age and sex distribution with the study group. None of the control group individuals had symptoms suggestive of influenza or coryza at the time of the study. As far as was practicable, the cases were matched for sex and age with a control subject. In the case group, the entire cohort reported symptoms affecting smell and in every case these symptoms had their onset either simultaneously with the onset of other symptoms of COVID-19 (pyrexia, coughing or dyspnea) or shortly thereafter. These olfactory symptoms had not been present in any of the case group prior to COVID-19 infection. There was a complete absence of olfactory complaints amongst the controls. Olfactory and gustatory testings were carried out by an examiner utilizing stringent safety standards and wearing full personal protective equipment. The results obtained in the case group were then compared with those obtained for the controls.

\section{Olfactory function testing}

The sense of smell was evaluated in all participants by means of the "Sniffin' Sticks" (Sniffin' Sticks, Burghart GmbH, Wedel, Germany) $[13,14]$ test battery, which tests psychological and physical aspects of olfactory function with felttipped pens. The various odours were presented as follows: the examiner took off the cap of each stick and presented it at a distance of around $1-2 \mathrm{~cm}$ away from the participant's nostrils. Sixteen commonly encountered odours were chosen. The participants had to choose from a list of four possible descriptive terms for each odour. Between each odour, a gap of at least $30 \mathrm{~s}$ was present as a precaution against olfactory desensitisation. The participants had control over how long 
they needed to decide on the odour presented. Scoring for the test depended on adding up the number of odours appropriately described by the test subjects $[13,14]$.

\section{Gustatory testing}

Gustatory testing involved identification of four solutions in water representing different types of taste: sweet, salty, sour and bitter. The sweet-tasting solution was $50 \%$ sucrose. Saltiness was tested using $0.9 \%$ saline solution. For sour taste, $4.2 \%$ vinegar was given. Finally, a $20 \%$ coffee mixture with no sweetening represented bitter taste [15]. The testing hours were during the morning to prevent any confounding effects from circadian alterations. The subjects had not eaten or drunk anything within the hour preceding the test. Each participant rinsed his or her mouth with distilled water first. They were supplied with a stopwatch in their right hand with which to record how long it took to identify the taste. Paper strips of identical dimensions were dipped into the previously prepared solutions and applied to the anterior twothirds of the lingual dorsum. The duration of time in seconds between strip placement and identification of the taste was recorded for each taste and for each subject. The participants used distilled water to flush out their mouths completely between the application of the paper strips $[15,16]$.

\section{Statistical analysis}

The IBM SPSS for Windows 21.0 application was used for all statistical analyses. Shapiro Wilk's normality test was used to determine the distribution of each variable. Chisquare testing was employed for the analysis of the various cross-tabulations produced (Group vs gender, group vs smell complaint, group vs taste complaint). In intergroup comparisons, the $t$ test (independent samples $t$ test) was used for samples independent of parametric tests, such as age. The Mann-Whitney $U$ test (non-parametric test) was used for the variables sweet, salty, sour and bitter. Number (as percentage), mean $( \pm \mathrm{SD})$ and median $(\mathrm{Q} 1 ; \mathrm{Q} 3)$ were used to summarize the data. A p value below 0.05 values was taken to indicate statistical significance.

\section{Results}

Eighty-one hospitalized patients with laboratory-confirmed SARS-CoV-2 infection represented the cases, whilst forty individuals in whom COVID-19 testing produced a negative result made up the controls. In the case group, 41 (50.6\%) of patients were male and $40(49.4 \%)$ were female, with a mean age of $54.16 \pm 16.98$ years (range 18-95). In the control group, $21(52.5 \%)$ of patients were male and $19(47.5 \%)$ of patient were female, whilst the mean age was $55 \pm 15.39$ years (range 18-90). The sex distribution within the cases and controls is given in Table 1. There was no difference in terms of sex distribution between the case and control groups (Chi-square $=0.38, p>0.05$ ). Furthermore, no difference was observed in terms of age distribution between the case and control groups $(t=-0.265, p=0.792, p>0.05)$.

In the case group, $50(61.7 \%)$ patients had complaints related to olfaction, whilst no individual in the control group complained of olfactory symptoms. The Sniffin' Sticks' test indicated that COVID-19 infection was associated with significant impairment of olfactory function, when results were compared with the data on the controls or with the previously published normative data. The distribution of olfactory symptoms in the case group differed at the level of statistical significance from the control group (Chi-square $=39.57$, $p<0.001)$. The distribution of olfactory symptoms in both the cases and controls is provided in Table 2.

Turning to gustatory abnormalities, within the case group, 22 individuals (27.2\%) had taste malfunction. Most of the patients stated that they had difficulties to identify the odours and tastes of the food they had consumed during their illness. A statistically significant difference was found in the distribution of gustatory abnormalities between cases and controls (Chi-square $=11.515 p=0.0069, p<0.001)$. Similarly, gustatory testing demonstrated marked abnormalities

Table 2 Distribution of complaint of smell

\begin{tabular}{lll}
\hline Group & \multicolumn{2}{l}{ Olfactory symptoms } \\
\cline { 2 - 3 } & Absent & Present \\
\hline Case group (count, \% within the group) & $31(38.3 \%)$ & $50(61.7 \%)$ \\
Control group (count, \% within the group) & $40(100 \%)$ & $0(0 \%)$ \\
Total (count, \% within combined groups) & $71(58.7 \%)$ & $50(41.3 \%)$ \\
\hline
\end{tabular}

Table 1 Gender and age distribution of study and control group

\begin{tabular}{llll}
\hline Group & Sex & \\
\cline { 2 - 4 } & Male & Female & Total \\
\hline Case group, (count, \% within group) & $41(50.6 \%)$ & $40(49.4 \%)$ & $81(100 \%)$ \\
Control group, (count, \% within group) & $21(52.5 \%)$ & $19(47.5 \%)$ & $40(100 \%)$ \\
Total (count, \% within combined groups) & $62(51.2 \%)$ & $59(48.8 \%)$ & $121(100 \%)$ \\
\hline
\end{tabular}


in the cases compared both to the study controls and when the results were compared with the published normative data. The distribution of gustatory abnormalities in the case and control groups is given in Table 3.

Fifty individuals (61.7\%) within the case group reported the loss of either olfactory or gustatory function, with 29 $(35.80 \%)$ reporting olfactory loss alone, 1 (1.23\%) gustatory loss alone, and 20 (24.69\%) both olfactory and gustatory loss.

Details of the results of olfactory and gustatory testing and the statistical significance of the results for the study are provided in Table 4. This table was created with Median Q1-Q3 and Mann-Whitney $U$ statistics. The results of these tests were generally in agreement.

\section{Discussion}

COVID-19 is the disease caused by the novel coronavirus SARS-CoV-2, which has resulted in a high level of fatalities across the entire globe [5, 6]. COVID-19 infections cover a range of possible presentations, from asymptomatic cases to moderate through severe disease. Pneumonia may or may not occur [9]. The study cohort contained greater numbers of males than females, which reflects the previously reported demographic and clinical character of the disease [17]. In

Table 3 Distribution of gustatory abnormalities

\begin{tabular}{lll}
\hline Group & \multicolumn{2}{l}{ Gustatory abnormality } \\
\cline { 2 - 3 } & Absent & Present \\
\hline Case group (count, \% within the group) & $59(72.8 \%)$ & $22(27.2 \%)$ \\
Control group (count, \% within the group) & $40(100 \%)$ & $0(0 \%)$ \\
Total (count, \% within the group) & $71(58.7 \%)$ & $22(18.2 \%)$ \\
\hline
\end{tabular}

our study, there was no difference in sex distribution between the case and control groups (Chi-square $=0.38 p>0.05$ ).

From the start of the COVID-19 pandemic, it has been proposed that acute onset olfactory $+/-$ gustatory malfunction may represent an early marker of probable infection [18-21]. Olfactory and gustatory malfunction may occur even in the absence of other symptoms suggestive of COVID-19. Although it is known that coronaviruses cause the common cold, many individuals presenting to ENT with a common cold accompanied by olfactory or gustatory malfunction are given a diagnosis of post-viral anosmia [21]. Nonetheless, despite histopathological studies indicating that direct trauma to the olfactory mucosa does take place, the precise point at which this occurs has not yet been established [22]. A longstanding abnormality of olfactory function has also been linked to the previous SARS-CoV pandemic, which occurred in 2004-2005. Abnormalities of olfactory and gustatory function may also be due to SARSCoV-2, the agent causing COVID-19 [23]. The present study's aim was to further elucidate the relationship between COVID-19 and olfactory or gustatory malfunction.

Numerous studies have now identified the most likely receptor the SARS-CoV-2 virus uses and hence may explain how infection occurs: the angiotensin-converting enzyme II (ACE2) receptor [24]. Thus, cell populations which express ACE2 at elevated levels (pulmonary, intestinal, cardiac and renal) will be most at risk from infection by the virus [25]. Xu et al. [24] looked at how the ACE2 receptor was expressed in the oral cavity by examining RNA transcripts. Lingual expression of ACE2 was higher than in the cheek or gingiva. This differential expression may partly account for the gustatory loss seen in SARS-CoV-2 infection. Our results indicate that ageusia occurred in $27.2 \%$ (22 individuals) of confirmed cases of COVID-19.

Moein et al. [26] propose that the low incidence of gustatory abnormalities in COVID-19 is more reasonably
Table 4 Comparison of results obtained in the case and control groups

\begin{tabular}{lllrlll}
\hline Smell and taste tests & Groups & $n$ & Median & Q1-Q3 & $\begin{array}{l}z \text { statistics } \\
\text { (Mann- }\end{array}$ & $p$ \\
& & & & & Whitney $U$ ) & \\
\hline Smell tests & Study group & 81 & 6.00 & $4.00-8.00$ & -7.29 & $<\mathbf{0 . 0 0 1 *}^{*}$ \\
Smell tests & Control group & 40 & 10.00 & $10.00-10.75$ & & 0.17 \\
Taste test: sweet & Study group & 81 & 7.40 & $4.70-17.50$ & -1.36 & \\
Taste test: sweet & Control group & 40 & 6.40 & $4.40-16.20$ & & 0.07 \\
Taste test: salty & Study group & 81 & 8.70 & $6.30-16.90$ & -1.83 & 0.052 \\
Taste test: salty & Control group & 40 & 6.90 & $5.30-15.53$ & & \\
Taste test: sour & Study group & 81 & 12.30 & $5.75-17.55$ & -1.94 & 0.28 \\
Taste test: sour & Control group & 40 & 7.25 & $5.10-16.48$ & & -1.08 \\
Taste test: bitter & Study group & 81 & 7.50 & $5.40-16.25$ & & \\
Taste test: bitter & Control group & 40 & 6.50 & $5.45-13.05$ & & \\
\hline
\end{tabular}

*Statistically significant 
attributed to traumatic injury to the olfactory system than to damage to the gustatory apparatus itself (such as the lingual papillae or the sensory fibres). Indeed, gustatory loss is virtually always accompanied by olfactory abnormalities, whether secondary to viral infection or some other cause. Our results indicated that 22 (27.2\%) of COVID-19 cases complained of alteration in taste. Ageusia was distributed differently between cases and controls, a result with statistical significance (Chi-square $=11.515 p=$ $0.0069, p<0.001)$. Likewise, gustatory testing in COVID19 cases indicated a marked degree of gustatory abnormality when compared with either the study control group or the normative data available for the tests.

It is rare to encounter dysgeusia as the sole presenting complaint, as it generally occurs in association with anosmia, as it does in COVID-19 cases. Klopfenstein et al. [27] report that anosmia and dysgeusia co-occurred $85 \%$ of the time and where a nasal discharge was present, in over $50 \%$ of cases. The results of the present study indicate an occurrence of either anosmia or dysgeusia in $61.7 \%$ of COVID-19 cases (50 individuals), and of anosmia alone in $35.8 \%$ (29 individuals). Dysgeusia without anosmia was only present in one case (1.23\%), whilst co-occurrence of the two symptoms was present in $24.69 \%$ ( 20 individuals). These results support the notion that mechanical blockage of the nose is not the cause of anosmia in COVID-19 cases.

Changes in the sense of smell in COVID-19 sufferers remain a matter of debate. The literature so far contains no comprehensive account regarding hyposmia. In late March 2020, the American Academy of Otolaryngology-Head and Neck Surgery announced that anosmia and dysgeusia had been identified in individuals who went on to be positive for COVID-19. They made the suggestion that anosmia and dysgeusia be included amongst the candidates used to screen for cases of the disorder [28]. An initial study examining hyposmia and dysgeusia has been carried out on in-patient cases of SARS-CoV-2 at Hospital Sacco (Milan, Italy) [29]. Amongst 59 individuals questioned about taste or smelling problems, $33.9 \%$ admitted to alteration in either taste or olfaction, whilst $18.6 \%$ complained of both symptoms. An Iranian preliminary survey has brought to light a peak incidence in de novo anosmia dating from after the period SARS-CoV-2 became an epidemic infection [21]. Anosmia is already attributed to a post-viral aetiology in approaching $40 \%$ of adult cases; hence, it would be reasonable to conclude that COVID-19 may also lead to this complication [30]. That SARS-CoV can result in the death of neurones in a mice model has already been proven experimentally. This occurred when the pathogen was able to gain access to the CNS via the olfactory epithelium [31]. Previous research has already ascertained expression of the ACE2 receptor in nervous tissue (glia and neurones) [32]. The present study found olfactory dysfunction in 50 (61.7\%) individuals, and taste dysfunction in $22(27.2 \%)$.

The precise mechanism by which SARS-CoV-2 infection produces anosmia is not yet known, since a complete objective assessment of olfaction is not currently feasible given the contagious nature of COVID-19, and the contraindications to endonasal endoscopy that exist in such cases [33]. Two potential mechanisms need to be considered: mechanical blockage of olfactory function secondary to inflammation, as seen in the olfactory cleft syndrome [34]; or neurotrauma produced when the olfactory epithelium and sensory neurones are destroyed by infection [33].

Anecdotal evidence favours considering hyposmia or hypogeusia as early indicators of SARS-CoV-2 infection before the clinical syndrome fully develops. At present, nonetheless, there is a lack of clarity regarding how far such symptoms are a risk factor for hospital admission, are a result of the virus or are just due to observer bias (i.e. seeing what it is expected will be seen). Establishing the temporal relationship between COVID-19 and hyposmia/ hypogeusia is essential if these symptoms are of value as markers of otherwise asymptomatic disease and hence as a way to identify contagious individuals.

Once an individual has recovered from SARS-CoV-2 infection, it is expected that one of two outcomes may occur: if the disorder is due to mechanical obstruction, complete resolution will occur; if, however, the problem is neurological, resolution is more complex. Up to now, there are insufficient data to know which the actual situation is. The present study would require extension over a longer period to provide data on resolution of hyposmia or hypogeusia. However, the evidence so far points to hyposmia and hypogeusia as indicators of SARS-CoV-2 infection. The present study, as far as we are aware, is the validated olfactory measures that confirm the prevalence of smell/taste loss.

The cases enrolled in this study were hospital in-patients at the time of assessment but data regarding their clinical outcome were not available to the researchers at the time of writing. The study needs to be extended to assess the duration of symptoms and their resolution. The study was undertaken when COVID-19 was pandemic and the use of advanced imaging and diagnostic modalities, such as endoscopy, paranasal sinus tomography and/or magnetic resonance imaging, was contraindicated due to the risk of cross-infection by the virus.

\section{Conclusion}

Olfactory and gustatory dysfunctions are strongly associated with SARS-CoV-2 infection. Hyposmia with or without hypogeusia is potentially a reliable indicator of latent COVID-19. The majority of cases up to the time of writing 
have featured temporary olfactory dysfunction with restoration of normal olfaction occurring from days to weeks after COVID-19 has passed. It is not yet clarified what percentage of cases are characterized by permanent olfactory dysfunction, and more detailed and long-term studies are needed in this regard. The results of this study support the contention that hyposmia or hypogeusia is important symptom to screen for in the context of identifying presymptomatic cases that may go on to infect others.

Funding This research did not receive any specific grant from funding agencies in the public, commercial or not-for-profit sectors.

\section{Compliance with ethical standards}

Conflict of interest The authors declare no conflict of interest.

\section{References}

1. Su S, Wong G, Shi W et al (2016) Epidemiology, genetic recombination, and pathogenesis of coronaviruses. Trends Microbiol 24(6):490-502. https://doi.org/10.1016/j.tim.2016.03.003

2. Drosten C, Günther S, Preiser W et al (2003) Identification of a novel coronavirus in patients with severe acute respiratory syndrome. N Engl J Med 348:1967-1976. https://doi.org/10.1056/ NEJMoa030747

3. Zaki AM, van Boheemen S, Bestebroer TM, Osterhaus AD, Fouchier RA (2012) Isolation of a novel coronavirus from a man with pneumonia in Saudi Arabia. N Engl J Med 367:1814-1820. https://doi.org/10.1056/NEJMoa1211721

4. Lu R, Zhao X, Li J et al (2020) Genomic characterisation and epidemiology of 2019 novel corona- virus: implications for virus origins and receptor binding. Lancet 395:565-574. https://doi. org/10.1016/S0140-6736(20)30251-8

5. Guan WJ, Ni ZY, Hu Y et al (2020) Clinical characteristics of coronavirus disease 2019 in China. N Engl J Med 382:1708-1720. https://doi.org/10.1056/NEJMoa2002032

6. Lechien JR, Chiesa-Estomba CM, De Siati DR et al (2020) Olfactory and gustatory dysfunctions as a clinical presentation of mildto-moderate forms of the coronavirus disease (COVID-19): a multicenter European study. Eur Arch Otorhinolaryngol. 2020:1-11. https://doi.org/10.1007/s00405-020-05965-1 (published online ahead of print, 2020 Apr 6)

7. Lai CC, Shih TP, Ko WC, Tang HJ, hSueh PR (2020) Severe acute respiratory syndrome coronavirus 2 (SARS-CoV-2) and coronavirus disease-2019 (COVID-19): the epidemic and the challenges. Int J Antimicrob Agents 17:105924. https://doi.org/10.1016/j.ijant imicag.2020.105924

8. Ceccarelli M, Berretta M, Venanzi Rullo E, Nunnari G, Cacopardo B (2020) Differences and similarities between Severe Acute Respiratory Syndrome (SARS)-CoronaVirus (CoV) and SARSCoV-2. Would a rose by another name smell as sweet? Eur Rev Med Pharmacol Sci 24(5):2781-2783. https://doi.org/10.26355/ eurrev_202003_20551

9. Dong X, Cao Y-Y, Lu X-X et al (2020) Eleven faces of coronavirus disease 2019. Allergy 00:1-11. https://doi.org/10.1111/all.14289

10. Tao C, Di W, Huilong C et al (2020) Clinical characteristics of 113 deceased patients with coronavirus disease 2019: retrospective study. BMJ 368:1091. https://doi.org/10.1136/bmj.m1091
11. Lee Y, Min P, Lee S, Kim SW (2020) Prevalence and duration of acute loss of smell or taste in COVID-19 patients. J Korean Med Sci 35(18):e174. https://doi.org/10.3346/jkms.2020.35.e174

12. World Health Organization. (2020) Clinical management of severe acute respiratory infection when Novel coronavirus ( $\mathrm{nCoV}$ ) infection is suspected: interim guidance. https://www.who.int/internalpublicationsdetail/clinical-management-of-severe-acuterespirator y-infection-when-novel-coronavirus-(ncov)-infection-is-suspe cted. Accessed 5 Feb 2020.

13. Hummel T, Sekinger B, Wolf SR, Pauli E, Kobal G (1997) "'Sniffin' Sticks': olfactory performance assessed by the combined testing of odor identification, odor discrimination and olfactory threshold. Chem Senses 22(1):39-52. https://doi.org/10.1093/ chemse/22.1.39

14. Altundağ A, Salihoglu M, Çayönü M, Cingi C, Tekeli H, Hummel T (2014) The effect of high altitude on olfactory functions. Eur Arch Otorhinolaryngol 271:615-618. https://doi.org/10.1007/ s00405-013-2823-3

15. Kale YS, Vibhute N, Belgaumi U et al (2019) Effect of using tobacco on taste perception. J Family Med Prim Care 8(8):2699_ 2702. https://doi.org/10.4103/jfmpc.jfmpc_457_19

16. Dyasanoor S, Abdul Khader NF (2016) Alteration in salivary properties and taste perception in OSMF. Contemp Clin Dent 7(2):146-152. https://doi.org/10.4103/0976-237X.183042

17. Fu L, Wang B, Yuan $\mathrm{T}$ et al (2020) Clinical characteristics of coronavirus disease 2019 (COVID-19) in China: a systematic review and meta-analysis. J Infect S0163-4453(20):30170-30175. https ://doi.org/10.1016/j.jinf.2020.03.041 (published online ahead of print, 2020 Apr 10)

18. Bagheri SHR, Asghari AM, Farhadi M et al (2020) Coincidence of COVID-19 epidemic and olfactory dysfunction outbreak. J Infect. https://doi.org/10.1016/j.jinf.2020.03.041

19. - Rabin RC. Lost sense of smell may be peculiar cluse to coronavirus infection. Web site Available at: https://www.nytim es.com/2020/03/22/health/coronavirus-symptoms-smell-taste.

20. - Stone J. There's an unexpected loss of smell and taste in coronavirus patients. Available at: https://www.forbes.com/sites/judys tone/2020/03/20/theres-an-unexpected-loss-of-smell-and-taste-incoronavirus-patients/\#5a90a8d51017. Published 2020.

21. Gilani S, Roditi R, Naraghi M (2020) COVID-19 and anosmia in Tehran, Iran. Med Hypotheses 141:109757. https://doi. org/10.1016/j.mehy.2020.109757 (published online ahead of print, 2020 Apr 23)

22. van Riel D, Verdijk R, Kuiken T (2015) The olfactory nerve: a shortcut for infuenza and other viral diseases into the central nervous system. J Pathol 235:277-287. https://doi.org/10.1002/ path. 4461

23. Hwang CS (2006) Olfactory neuropathy in severe acute respiratory syndrome: report of A case. Acta neurologica Taiwanica 15(1):26-28 (PMID: 16599281)

24. Xu H, Zhong L, Deng J et al (2020) High expression of ACE2 receptor of 2019-nCoV on the epithelial cells of oral mucosa. Int J Oral Sci. https://doi.org/10.1038/s41368-020-0074-x (Article number: 8)

25. Zou X, Chen K, Zou J, Han P, Hao J, Han Z (2020) The single-cell RNA-seq data analysis on the receptor ACE2 expression reveals the potential risk of different human organs vulnerable to Wuhan 2019-nCoV infection. Front Med. https://doi.org/10.1007/s1168 4-020-0754-0

26. Moein ST, Hashemian SMR, Mansourafshar B, Khorram-Tousi A, Tabarsi P, Doty RL (2020) Smell dysfunction: a biomarker for COVID-19. Int Forum Allergy Rhinol. https://doi.org/10.1002/ alr.22587

27. Klopfenstein T, Kadiane-Oussou NJ, Toko L et al (2020) Features of anosmia in COVID-19. Medecine et Maladies Infectieuses. https://doi.org/10.1016/j.medmal.2020.04.006 
28. - Web site. Available at: https://www.entnet.org/content/aao-hnsanosmia-hyposmia-anddysgeusia-symptoms-coronavirus-disease. Accessed 25 Mar 2020.

29. Giacomelli A, Pezzati L, Conti F et al (2020) Self-reported olfactory and taste disorders in SARS-CoV-2 patients: a cross-sectional study. Clin Infect Dis. https://doi.org/10.1093/cid/ciaa330 (published online ahead of print 2020)

30. Hummel T, Whitcroft KL, Andrews P, Altundag A, Cinghi C et al (2016) Position paper on olfactory dysfunction. Rhinology 56(1):1-30. https://doi.org/10.4193/Rhin16.248

31. Netland J, Meyerholz DK, Moore S, Cassell M, Perlman S (2008) Severe acute respiratory syndrome coronavirus infection causes neuronal death in the absence of encephalitis in mice transgenic for human ACE2. J Virol 82(15):7264-7275. https://doi. org/10.1128/JVI.00737-08

32. Baig AM, Khaleeq A, Ali U, Syeda H (2020) Evidence of the COVID-19 virus targeting the CNS: tissue distribution, host-virus interaction, and proposed neurotropic mechanisms. ACS Chem Neurosci 11(7):995-998. https://doi.org/10.1021/acschemneu ro.0c00122

33. Gane SB, Kelly C, Hopkins C (2020) Isolated sudden onset anosmia in COVID-19 infection. A novel syndrome? Rhinology. https ://doi.org/10.4193/Rhin20.114

34. Trotier D, Bensimon JL, Herman P, Tran Ba Huy P, Døving KB, Eloit C (2006) Inflammatory obstruction of the olfactor y clefts and olfactory loss in humans: a new syndrome? Chem Senses 32(3):285-292. https://doi.org/10.1093/chemse/bj1057

Publisher's Note Springer Nature remains neutral with regard to jurisdictional claims in published maps and institutional affiliations. 